\title{
On the rate equation of nucleation and the concept of active sites in electrodeposition
}

\author{
J.H.O.J. Wijenberg, W.H. Mulder, M. Sluyters-Rehbach and J.H. Sluyters * \\ Van 't Hoff Laboratory, State University of Utrecht, Padualaan 8, 3584 CH Utrecht (The Netherlands)
}

(Received 26 April 1988; in revised form 20 June 1988)

\begin{abstract}
On the basis of the assumption that on a homogeneous surface area a probability $0<P(E, c)<1$ for nucleation can be defined which increases with the monomer concentration $c$ and is also dependent on the potential $E$, it is shown both by a schematic computer simulation and by an approximate analytical derivation that necessarily the number of nuclei has to attain a limiting value for long times, without having to resort to the existence of active sites. It follows that this limiting number arises from a concurrence between the probability of nucleation and the processes that accompany the growth of a nucleus and that decrease supersaturation.
\end{abstract}

\section{LIST OF SYMBOI S}

$A \quad$ nucleation rate per active site, $\mathrm{s}^{-1}$

$c(r, t)$ monomer concentration at a radial distance $r$ from the centre of a lone nucleus at time $t, \mathrm{~mol} \mathrm{~cm}^{-3}$

$c^{*} \quad$ monomer bulk concentration, $\mathrm{mol} \mathrm{cm}^{-3}$

$D \quad$ diffusion coefficient, $\mathrm{cm}^{2} \mathrm{~s}^{-1}$

$k \quad$ growth constant of exclusion zone, $\mathrm{cm} \mathrm{s}^{-1}$

$N(t) \quad$ number density of nuclei at time $t, \mathrm{~cm}^{-2}$

$N_{0} \quad$ number density of active sites, $\mathrm{cm}^{-2}$

$P(E, c)$ nucleation rate probability as a function of potential $E$ and concentration $c, \mathrm{~cm}^{-2} \mathrm{~s}^{-1}$

$R(t) \quad$ radius of a nucleus at time $t, \mathrm{~cm}$

$\underline{R}(t, u)$ radius of exclusion zone at time $t$ belonging to a lone nucleus, which is born at time $u, \mathrm{~cm}$

\footnotetext{
ऋ To whom correspondence should be addressed.
} 
$S \quad$ fractional surface area excluded for further nucleation

$S$ Avrami's extended area

$\alpha \quad=\pi k^{2}$

$\beta \quad=(\mathrm{d} N(t) / \mathrm{d} t)_{t=0}, \mathrm{~cm}^{-2} \mathrm{~s}^{-1}$

$\gamma \quad$ factor with which $P(E, c)$ decreases because of depletion

$\rho / M \quad$ molar density, $\mathrm{mol} \mathrm{cm}^{-3}$

\section{(1) INTRODUCTION}

A large body of literature exists dealing with the mathematical description of the electrodeposition of some material on a foreign substrate [1-33]. These theories, at present, consist mostly of combining theories on nucleation, growth of the nuclei under diffusion and/or activation control, overlap of the growing nuclei and/or the diffusion zones that surround them, ohmic effects, etc. From these partial processes nucleation is the crucial one because it largely determines how the overall process will proceed.

Generally, the nucleation process is supposed to be stochastic in time and to occur only at certain locations randomly distributed on the electrode surface, named active sites [1-4,6,15-32]. Although speculations on the nature of active sites do occur in the literature $[1-3,30]$ and although there is clear evidence that nucleation occurs preferentially on scratches in the electrode surface [18], the question of whether active sites are a physical reality also on well-prepared supposedly homogeneous electrode surfaces seems to be unsettled. On the other hand, the number of sites, i.e. the quantity $N_{0}$ in the generally accepted nucleation law, $\mathrm{d} N / \mathrm{d} t=A\left(N_{0}-\right.$ $N$ ), on any surface is found to be finite in practice, although dependent on, for example, the electrode potential and electrode pretreatment $[2,3,6,15-17,19,20,23$, $25,26,30]$.

In particular, the dependence on the electrode potential has remained puzzling and has been explained assuming the site to occur in a wide and continuous spectrum of activities, each site being active only beyond some value of the electrode potential $[1-3,6,19,22]$. Also it has been argued that sites will become inactive if the concentration of the electroactive species in their vicinity has become low because of the consumption of the species by neighbouring growing sites (ingestion effect $[3,5,8,11,15,18,20-23,30-32])$.

In this paper we show that the occurrence of a finite number of nuclei can also be explained on the plausible assumption of the existence of a probability $P$ (in $\mathrm{cm}^{-2}$ $\mathrm{s}^{-1}$ ) for the birth of a nucleus, which increases with the concentration of the electroactive species, thus abandoning the concept of the active site.

\section{(II) A COMPUTER SIMULATION OF NUCLEATION WITH INGESTION}

The electrode surface is supposed to be completely homogeneous. All over the surface the probability for a nucleus to arise is defined by $0<P(E, c)<1$, which is a function of both the potential $E$ and the concentration of the electroactive species 
$c$ via some rate equation. Then if a nucleus is born and has started to grow, in its neighbourhood the value of $P(E, c)$ will decrease because of the build up of a region of decreased concentration, the depletion zone. The form of this zone in the case where growth of the nucleus is governed by hemispherical diffusion and at high overpotential (surface concentration of the nucleus zero) has been shown to be [6,32]

$c(r, t)=c^{*}[1-R(t) / r] \operatorname{erfc}\left[\{r-R(t)\} / 2(D t)^{1 / 2}\right] \quad$ for $r \geq R(t)$

where $R(t)=\left(2 D c^{*} M / \rho\right)^{1 / 2} t^{1 / 2}, c^{*}$ is the bulk concentration, $D$ the diffusion cocfficient of the electroactive species and $\rho / M$ the molar density of the deposit. Equation (1) describes how the depletion propagates both in time and in distance from the nucleus.

Here, in order to keep both the discussion and the computer program simple, for the present the build up of the zone around a nucleus will be introduced very schematically, although the essence of the method is retained. For the same reason the simulation will be made in one dimension only. Along this dimension the "surface" is divided into 80 discrete elements to make possible the simulation that runs as follows.

The decision whether in an element a nucleus is born is made via a random number generation. At the start of the program, all elements have the same probability $P$ of generating a nucleus. Then in the second run all elements adjacent to a successful element are given a lower probability $\gamma P$. This power law decrease goes on, but we desisted from decreasing the probability beyond the next nearest elements. Of course, in every run new nuclei are allowed to be born as a result of decisions made by the random number generator on the basis of the prevailing value of the probability. In order to avoid edge effects the ends of the "surface" were connected.

In order to give a visual explanation, in Table 1 as an example we report the

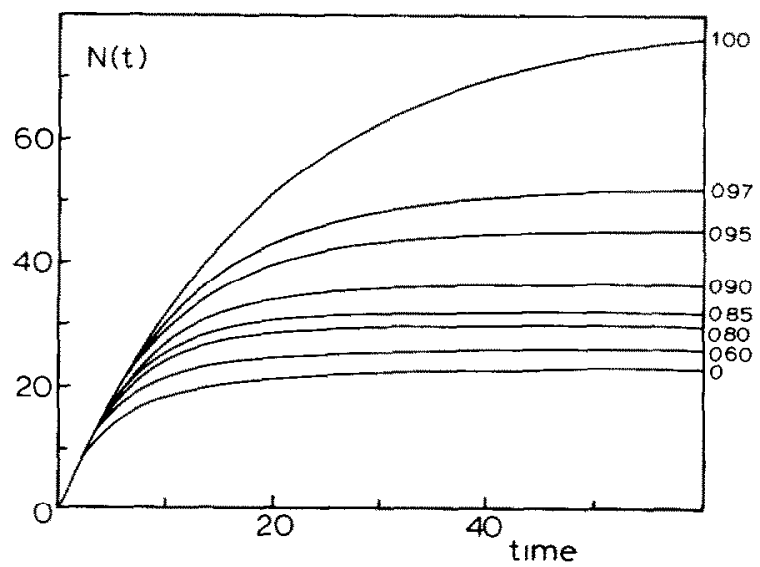

Fig. 1. Development of the nucleus density with time obtained with $P=0.05$ for some indicated values of $\gamma$, averaged over 500 simulations. 
development of the probabilities along a part of the "surface". Also we indicate each of the successful nucleations with an $\mathrm{N}$.

It will be clear that in this way the development of the number of nuclei with time can be obtained. To remove the stochastic noise from the results, 500 of such simulations were averaged. These averaged results are reported in Fig. 1 for eight indicated values of the parameter $\gamma$, and $P=0.05$.

A value $\gamma=1$ indicates no depletion and will occur if mass transport is infinitely fast. Obviously only then the monomolecular development of the nucleus density will be observed according to the well-known rate equation

$N(t)=N_{0}[1-\exp (-A t)]$

For all other values of $\gamma$, the rate equation differs from eqn. (2). Another interesting result, reproduced in Fig. 2, is the limiting value $N(\infty)$ that occurs after a large number of time increments (here 300). Most surprisingly, the limiting number of nuclei is strongly dependent on the parameter $\gamma$ at $\gamma$ close to unity and is rather independent of $\gamma$ for $\gamma<0.8$. From its definition it will be clear that a high value of $\gamma$, i.e. close to unity, corresponds to a high mass-transfer rate. So, under such experimental conditions the density of the nuclei will be strongly dependent on the rate of mass transfer, i.e. when depletion is minor.

Finally, we report the development of $N(t)$ with time for some values of $P$ at constant $\gamma=0.9$ in Fig. 3.

A variation of $P$ experimentally can be brought about by varying the electrode potential. Evidently from Fig. 3 the potential dependence of the limiting value $N(\infty)$ can be understood. A second corollary of Fig. 3 is that at constant rate of mass transfer a causal relationship should exist between $N(\infty)$ and $P$, or to put it in usual terminology, between the active site density and the nucleation rate constant.

\section{TABLE 1}

Development of the probabilities along a part of the "surface". Successful nucleations are indicated with

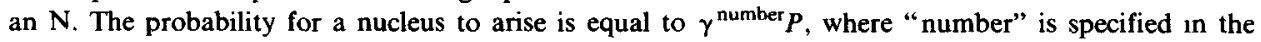

\begin{tabular}{rrllllllllllll}
\hline 0 & 0 & 0 & 0 & 0 & 0 & 0 & 0 & 0 & 0 & 0 & 0 & 0 & 0 \\
0 & 0 & 0 & 0 & 0 & 0 & $\mathrm{~N}$ & 0 & $\mathrm{~N}$ & 0 & 0 & 0 & 0 & 0 \\
0 & 0 & 0 & 0 & 0 & 1 & $\mathrm{~N}$ & 2 & $\mathrm{~N}$ & 1 & 0 & 0 & $\mathrm{~N}$ & 0 \\
0 & 0 & 0 & 0 & 1 & 2 & $\mathrm{~N}$ & 4 & $\mathrm{~N}$ & 2 & 1 & 1 & $\mathrm{~N}$ & 1 \\
0 & 0 & 0 & 0 & 2 & 3 & $\mathrm{~N}$ & 6 & $\mathrm{~N}$ & 3 & 3 & 2 & $\mathrm{~N}$ & 2 \\
0 & 0 & 0 & $\mathrm{~N}$ & 3 & 4 & $\mathrm{~N}$ & 8 & $\mathrm{~N}$ & 4 & 5 & 3 & $\mathrm{~N}$ & 3 \\
0 & 0 & 1 & $\mathrm{~N}$ & 5 & 5 & $\mathrm{~N}$ & 10 & $\mathrm{~N}$ & 5 & 7 & 4 & $\mathrm{~N}$ & 4 \\
0 & 1 & 2 & $\mathrm{~N}$ & 7 & 7 & $\mathrm{~N}$ & 12 & $\mathrm{~N}$ & 6 & 9 & 5 & $\mathrm{~N}$ & 5 \\
0 & 2 & 3 & $\mathrm{~N}$ & 9 & 9 & $\mathrm{~N}$ & 14 & $\mathrm{~N}$ & 7 & 11 & 6 & $\mathrm{~N}$ & 7 \\
0 & 3 & 4 & $\mathrm{~N}$ & 11 & 11 & $\mathrm{~N}$ & 16 & $\mathrm{~N}$ & 8 & 13 & 7 & $\mathrm{~N}$ & 9 \\
0 & 4 & 5 & $\mathrm{~N}$ & 13 & 13 & $\mathrm{~N}$ & 18 & $\mathrm{~N}$ & 9 & 15 & 8 & $\mathrm{~N}$ & 11 \\
0 & 5 & 6 & $\mathrm{~N}$ & 15 & 15 & $\mathrm{~N}$ & 20 & $\mathrm{~N}$ & 10 & 17 & 9 & $\mathrm{~N}$ & 13 \\
0 & 6 & 7 & $\mathrm{~N}$ & 17 & 17 & $\mathrm{~N}$ & 22 & $\mathrm{~N}$ & 11 & 19 & $\mathrm{~N}$ & $\mathrm{~N}$ & 15 \\
0 & 7 & 8 & $\mathrm{~N}$ & 19 & 19 & $\mathrm{~N}$ & 24 & $\mathrm{~N}$ & 12 & 22 & $\mathrm{~N}$ & $\mathrm{~N}$ & 17 \\
\hline
\end{tabular}




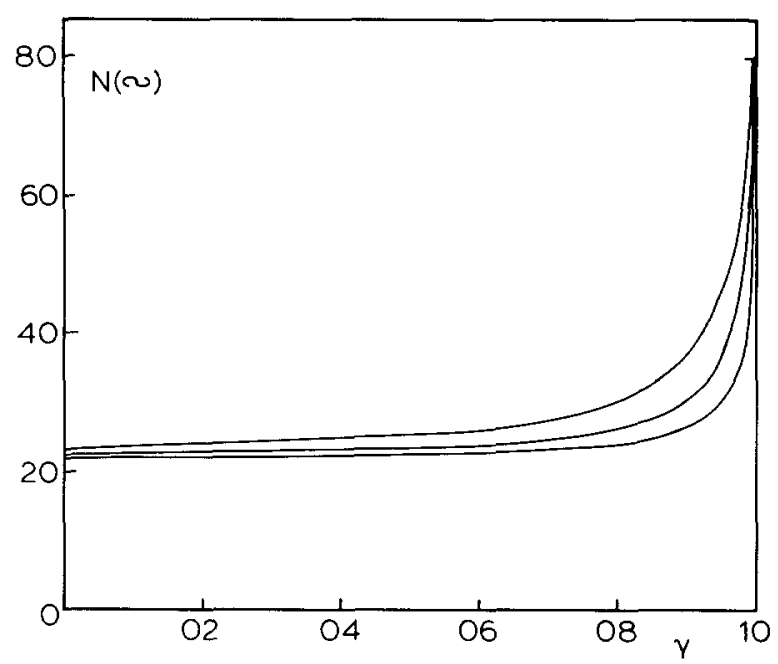

Fig. 2. Limiting values $N(\infty)$ of the nucleus density as a function of the parameter $\gamma$, obtained with (from top to bottom) $P=0.05,0.025$ and 0.0125 , respectively.

It might be argued that dividing the surface into a finite number of elements is identical to saying that there is the same number of active sites. The problem arises only because of the necessity, for the sake of the simulation, to make the nucleation process discrete in time and space. The way out is to realize that in reality the number of elements should be taken as infinite and the probabilities accordingly small. Then for $\gamma=1$ the value of $N(\infty)$ will be infinite, as is to be expected for a

table. $P$ was taken equal to 0.05 and $\gamma$ to 0.9 . The rows show the situation after a number $(0$ to 13$)$ of time intervals have elapsed

\begin{tabular}{llllllllllllll}
\hline 0 & 0 & 0 & 0 & 0 & 0 & 0 & 0 & 0 & 0 & 0 & 0 & 0 & 0 \\
0 & 0 & 0 & 0 & 0 & 0 & 0 & 0 & 0 & 0 & 0 & 0 & 0 & 0 \\
0 & 0 & 0 & 0 & 0 & 0 & 0 & 0 & 0 & 0 & 0 & 0 & 0 & 0 \\
0 & 0 & 0 & 0 & 0 & 0 & 0 & 0 & 0 & 0 & 0 & 0 & 0 & 0 \\
1 & 0 & 0 & 0 & 0 & 0 & 0 & 0 & 0 & 0 & 0 & 0 & 0 & 0 \\
2 & 0 & 0 & 0 & 0 & 0 & 0 & 0 & 0 & 0 & 0 & 0 & 0 & 0 \\
3 & 0 & 0 & 0 & 0 & 0 & 0 & 0 & 0 & 0 & 0 & 0 & 0 & 0 \\
$\mathbf{N}$ & 0 & 0 & 0 & 0 & 0 & 0 & 0 & 0 & 0 & 0 & $\mathbf{N}$ & 0 & 0 \\
$\mathbf{N}$ & 1 & 0 & 0 & 0 & 0 & 0 & 0 & 0 & 0 & 1 & $\mathbf{N}$ & 1 & 0 \\
$\mathrm{~N}$ & 2 & 1 & 0 & $\mathbf{N}$ & 0 & 0 & 0 & 0 & 1 & 2 & $\mathbf{N}$ & 2 & 1 \\
$\mathbf{N}$ & 3 & 2 & 1 & $\mathbf{N}$ & $\mathbf{N}$ & 0 & 0 & 0 & 2 & 3 & $\mathbf{N}$ & 3 & 2 \\
$\mathbf{N}$ & 4 & 4 & 2 & $\mathbf{N}$ & $\mathbf{N}$ & 2 & 0 & 0 & 3 & 4 & $\mathbf{N}$ & 4 & 3 \\
$\mathbf{N}$ & 5 & 6 & $\mathbf{N}$ & $\mathbf{N}$ & $\mathbf{N}$ & 4 & 1 & 0 & 4 & 5 & $\mathbf{N}$ & 5 & 4 \\
$\mathbf{N}$ & 6 & 9 & $\mathbf{N}$ & $\mathbf{N}$ & $\mathbf{N}$ & 6 & 2 & 0 & 5 & 6 & $\mathbf{N}$ & 6 & 5 \\
\hline
\end{tabular}




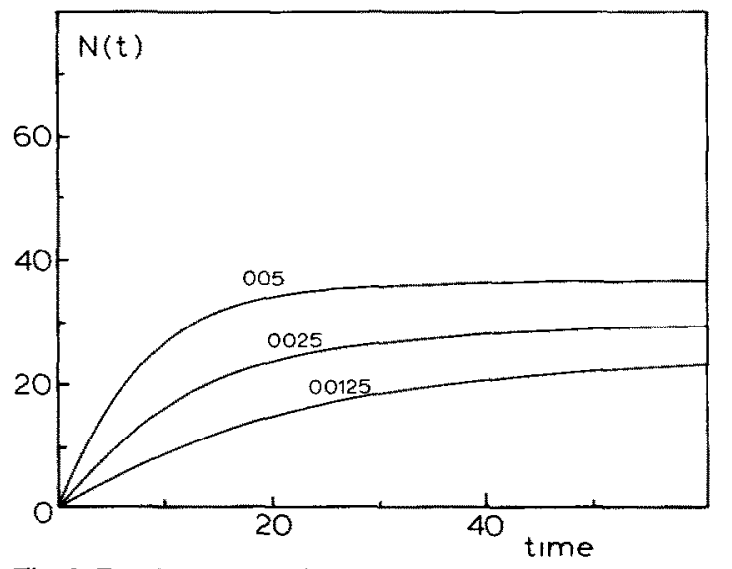

Fig. 3. Development of the nucleus density with time calculated with $\gamma=0.9$ for some indicated values of $P$, averaged over 500 simulations.

homogeneous surface, and a finite number of nuclei will occur in reality only because mass transfer can never be infinitely fast.

Although the adaptation of the present simulation procedure to real systems will be more difficult (e.g. performing it on the basis of eqn. 1, including the ohmic drop and implementing it with a real relationship between $P$ and $c$ and possibly between $P$ and cluster size [33]), we think it is legitimate to draw some qualitative conclusions. A relationship is expected to exist between the limiting number of nuclei $N(\infty)$, the probability $P$ and the mass-transfer coefficient $\gamma$. Also the existence of active sites is not an inevitable assumption to explain the occurrence of a limiting number of nuclei for long times. It should be realized that the factor $\gamma$ could comprise all other effects that tend to decrease supersaturation, like the effect of ohmic drop and rise of temperature if the growth of a nucleus is exothermic.

An analogous simulation in two dimensions yielded graphs quite similar to Figs. $1-3$. This program is most suitable for an on-line demonstration and is available on request.

\section{(III) AN ANALYTICAL EXPRESSION FOR THE NUCLEATION RATE}

The simulation in the preceding section could be made more realistic by implementing it with the mathematics of mass transfer, the kinetics of the nucleation process yielding $P(E, c)$, the kinetics of growth and the ohmic drop effect. A completely rigorous derivation of an expression describing the development of the number of nuclei with time $N(t)$ seems to be infeasible. However, an acceptable compromise (based on the Avrami theorem applied in a way analogous to the current theories on the effect of ingestion of active sites by the growing and ovcrlapping depletion zones) is possible in the following way, restricting the 
discussion to diffusion-controlled hemispherical growth of the nuclei. Then it can be postulated that, after the nucleation process has started at $t=0$, around every growing nucleus born at time $t=u$ an effective exclusion zone develops with radius $\underline{R}(t, u)=k(t-u)^{1 / 2}[5,15,20,23,31,32]$, where $k$ stands for the growth constant of the zone within which no nucleation is possible. So, every nucleus is the centre of a circle with surface arca $\pi \underline{R}^{2}(t, u)$, excluded from nucleation. Of course, in reality the nucleation probability will vary continuously from the value belonging to the bulk monomer concentration to that belonging to the concentration at the surface of the nucleus, dictated by the Nernst equation for a reversible system. The extended surface area, i.e. the surface of all the exclusion circles including those which originated from the ingested nuclei (often denoted in the literature as phantoms or ghosts) will be, with the initial condition $N(0)=0$ :

$$
\begin{aligned}
S_{x} & =\pi \int_{0}^{t}\left(\frac{\mathrm{d} N}{\mathrm{~d} t}\right)_{t=0} \underline{R}^{2}(t, u) \mathrm{d} u=\pi k^{2}\left(\frac{\mathrm{d} N}{\mathrm{~d} t}\right)_{t=0} \int_{0}^{t}(t-u) \mathrm{d} u \\
& =\frac{1}{2} \pi k^{2}\left(\frac{\mathrm{d} N}{\mathrm{~d} t}\right)_{t=0} t^{2}
\end{aligned}
$$

This area should be corrected for overlap of the exclusion zones with the Avrami equation, which states that the fractional surface area corrected for overlap, $S$, is related to $S_{x}$ according to

$S=1-\exp \left(-S_{x}\right)$

The nucleation rate is proportional to the free fractional surface area $(1-S)$, whence

$$
\frac{\mathrm{d} N}{\mathrm{~d} t}=\left(\frac{\mathrm{d} N}{\mathrm{~d} t}\right)_{t=0} \exp \left(-\frac{1}{2} \pi k^{2}\left(\frac{\mathrm{d} N}{\mathrm{~d} t}\right)_{t=0} t^{2}\right)=\beta \exp \left(-\frac{1}{2} \alpha \beta t^{2}\right)
$$

Here, $\alpha$ and $\beta$ have been introduced for convenience. Solving this differential equation gives

$N(t)=\left(\frac{\pi \beta}{2 \alpha}\right)^{1 / 2} \operatorname{erf}\left[\left(\frac{1}{2} \alpha \beta\right)^{1 / 2} t\right]$

For small values of $\left(\frac{1}{2} \alpha \beta\right)^{1 / 2}$, eqn. (6) reduces to

$$
N(t)=\beta t=\left(\frac{\mathrm{d} N}{\mathrm{~d} t}\right)_{t=0} t
$$

describing that part of the time domain that is usually referred to as the domain of progressive nucleation. Also the so-called "instantaneous limit" is described by eqn. (6), namely if $\left(\frac{1}{2} \alpha \beta\right)^{1 / 2} t$ is large:

$$
N(t)=(\pi \beta / 2 \alpha)^{1 / 2}
$$

In Fig. $4, N(t) /(\pi \beta / 2 \alpha)^{1 / 2}$ has been plotted vs. $(\alpha \beta / 2)^{1 / 2} t$. For long times $N(t)$ has the limiting value $N(\infty)=(\pi \beta / 2 \alpha)^{1 / 2}$. This means that the final number of nuclei will be large if the initial nucleation rate is high and/or the development of 


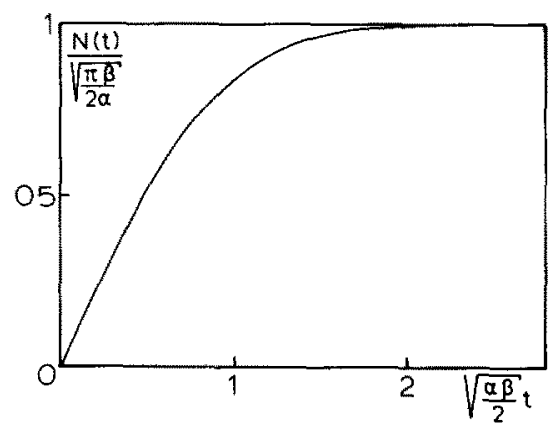

Fig. 4. Normalized plot of the development of nucleus density with time (cf. eqn. 6).

the exclusion zones is slow. The final number will be reached sooner if the initial nucleation rate is high and/or the exclusion zones develop rapidly. All this is in qualitative accordance with the results of the simulation procedure presented in Section (II).

\section{(IV) DISCUSSION}

Both the simulation and the analytical derivation lead to the conclusion that if the interface is assumed to be homogeneous as for nucleation, the two effects that counteract the supersaturation in the neighbourhood of growing nuclei, i.e. decrease of monomer concentration and ohmic drop, necessarily lead to a finite value of the nucleus density. Consequently the assumption of the existence of locations on the interface that are specially suitable for nucleation to occur, so-called active sites, is unnecessary. Of course, this does not mean that all practical surfaces are homogeneous and that active spots cannot occur, only that their use in theories should be restricted to those cases where their presence for some reason is to be considered realistic.

Electrodeposition is quite a complex process, made up of many partial processes that proceed simultaneously. In order to arrive at surveyable and tractable mathematical descriptions it is tempting in a derivation to restrict ourselves to only one or two of those partial processes and then to pose that for such a derivation always some time domain in the transient exists in which the derived behaviour will apply. This is a risky procedure and seems to have led to misinterpretations of experimental results [31].

Likewise, it is customary to assume that the ingestion effect becomes effective only in a later stage of electrodeposition. This idea was most probably nourished by the idea that the active sites are situated relatively far apart. We expect that with the views developed in this paper some further integration of the partial processes in electrocrystallization has been acquired.

As a conclusion we state that the ingestion effect should be regarded as a basic aspect of the nucleation process rather than as a complication to it. In cases where 
the ingestion occurs because of a temperature rise in the vicinity of a growing nucleus due to exothermic growth in a medium of low thermal conductivity these views may also apply to (non-electrochemical) phase transitions in a condensed phase.

\section{(V) COMPARISON WITH THE LITERATURE}

Many treatments on electrochemical nucleation and growth have been published, many of which include the ingestion effect. All of them seem in some way to assume the existence of sites randomly distributed on the surface. Physically this means that the surface is supposed to be inhomogeneous as for nucleation for some, generally unknown, reason.

On a crystal surface there can be a finite number of dislocations which could act as a site and then the active site model will be applicable. On the other hand, in the case of a practical electrode material so many imperfections could exist that the electrode surface might as well be treated as homogeneous. As a consequence, then the present treatment (after further elaboration) is to be preferred because it leads to an understanding of the potential dependence of the apparent number of active sites and to the use of a better rate equation for nucleation.

There is good experimental evidence that the probability for nucleation $P(E, c)$ is an existing property of an electrode/electrolyte interface, for it is closely related to the function $P$ which has been obtained experimentally on screw-dislocation-free silver surfaces by Obretenov et al. [13] in their mononuclear two-dimensional growth experiments on the electrochemical reduction of silver ions.

Similar experiments have been described by Gunawardena et al. [10] for a foreign substrate, from which the probability $P(E, c)$ for a nucleation to occur in unit time and at unit surface area can be obtained directly.

\section{ACKNOWLEDGEMENTS}

These investigations were supported in part by The Netherlands' Foundation for Chemical Research (SON) with financial aid from The Netherlands' Technology Foundation.

\section{REFERENCES}

1 S. Toschev and B. Mutaftschiew, Electrochim. Acta, 9 (1964) 1203.

2 R. Kaischew and B. Mutaftschiew, Electrochim. Acta, 10 (1965) 643.

3 I. Markov and D. Kashchiev, J. Cryst. Growth, 16 (1972) 170.

4 S.K. Rangarajan, J. Electroanal. Chem., 46 (1973) 119.

5 I. Markov, A. Boynov and S. Toschev, Electrochim. Acta, 18 (1973) 377.

6 G.J. Hills, D.J. Schiffrin and J. Thompson, Electrochim. Acta, 19 (1974) 657.

7 H. Angerstein-Kozlowska, B.E. Conway and J. Klinger, J. Electroanal. Chem., 87 (1978) 301.

8 A. Milchev, E. Vassileva and V. Kertov, J. Electroanal. Chem., 107 (1980) 323.

9 S. Fletcher and A. Smith, Electrochim. Acta, 25 (1980) 1019. 
10 G.A. Gunawardena, G.J. Hills and B. Scharifker, J. Electroanal. Chem., 130 (1981) 99.

11 E. Bosco and S.K. Rangarajan, J. Chem. Soc., Faraday Trans. 1, 77 (1981) 483.

12 V. Bostanov, W. Obretenov, G. Staikov, D.K. Roe and E. Budevski, J. Cryst. Growth, 52 (1981) 761.

13 W. Obretenov, V. Bostanov and V. Popov, J. Electroanal. Chem., 132 (1982) 273.

14 A. Milchev, B. Scharifker and G. Hills, J. Electroanal. Chem., 132 (1982) 277.

15 G. Gunawardena, G. Hills, I. Montenegro and B. Scharifker, J. Electroanal. Chem., 138 (1982) 225.

16 G. Gunawardena, G. Hills and I. Montenegro, J. Electroanal. Chem., 138 (1982) 241.

17 G. Gunawardena, G. Hills, I. Montenegro and B. Scharifker, J. Electroanal. Chem., 138 (1982) 255.

18 M.Y. Abyaneh, Electrochim. Acta, 27 (1982) 1329.

19 S. Fletcher and T. Lwin, Electrochim. Acta, 28 (1983) 237.

20 B. Scharifker and G. Hills, Electrochim. Acta, 28 (1983) 879.

21 E. Trevisan-Souteyrand, G. Maurin and D. Mercier, J. Electroanal. Chem., 161 (1984) 17.

22 R.L. Deutscher and S. Fletcher, J. Electroanal. Chem., 164 (1984) 1.

23 B.R. Scharifker and J. Mostany, J. Electroanal. Chem., 177 (1984) 13.

24 J. Mostany, J. Mozota and B.R. Scharifker, J. Electroanal. Chem., 177 (1984) 25.

25 G. Gunawardena, G. Hills and I. Montenegro, J. Electroanal. Chem., 184 (1985) 357.

26 G. Gunawardena, G. Hills and I. Montenegro, J. Electruanal. Chem., 184 (1985) 371.

27 A. Milchev, Electrochim. Acta, 30 (1985) 125.

28 V. Tsakova and A. Milchev, J. Electroanal. Chem., 197 (1986) 359.

29 A. Milchev, Electrochim. Acta, 31 (1986) 977 .

30 V. Tsakova and A. Milchev, J. Electroanal. Chem., 235 (1987) 237.

31 M. Sluyters-Rehbach, J.H.O.J. Wijenberg, E. Bosco and J.H. Sluyters, J. Electroanal. Chem., 236 (1987) 1.

32 R.L. Deutscher and S. Fletcher, J. Electroanal. Chem., 239 (1988) 17.

33 J.H. Sluyters, E. Bosco and M. Sluyters-Rehbach, J. Electroanal. Chem., 241 (1988) 79. 